\title{
Intérêts et opinions de Charles De Marguerye
} (1758-1840)

\section{Didier Michel}

\section{(2) OpenEdition \\ 1 Journals}

Édition électronique

URL : https://journals.openedition.org/ahrf/2310

DOI : 10.4000/ahrf.2310

ISSN : 1952-403X

\section{Éditeur :}

Armand Colin, Société des études robespierristes

\section{Édition imprimée}

Date de publication : 15 mars 2006

ISSN : 0003-4436

\section{Référence électronique}

Didier Michel, «Intérêts et opinions de Charles De Marguerye (1758-1840) », Annales historiques de la Révolution française [En ligne], 343 | janvier-mars 2006, mis en ligne le 22 septembre 2009, consulté le 24 avril 2022. URL : http://journals.openedition.org/ahrf/2310; DOI : https://doi.org/10.4000/ahrf. 2310

Ce document a été généré automatiquement le 24 avril 2022.

Tous droits réservés 


\title{
Intérêts et opinions de Charles De Marguerye (1758-1840)
}

\author{
Didier Michel
}

1 Travaillant sur un corpus de lettres reçues entre 1781 et 1792 par un lieutenant d'infanterie, le chevalier Blondel de Nouainville, la particularité des cinq lettres écrites en 1791 par le comte de Marguerye nous est vite apparue ${ }^{1}$. Ce personnage obscur ${ }^{2}$ y formule des opinions politiques, nous l'ayant fait percevoir d'emblée comme un noble libéral ${ }^{3}$. Cette qualification quelque peu impressionniste nécessite d'être précisée, encore qu'il soit généralement difficile de classer Marguerye dans des catégories prédéfinies. C'est à tout le moins sur le thème de la modération qu'il va falloir composer.

2 En effet, à suivre l'analyse de Georges Benrekassa, «entre l'automne 1790 et le printemps 1791, on trouve la trace d'une opération de séparation décisive entre la "vertu" de modération et l'action politique des "modérateurs" " ${ }^{4}$. Aussi les opinions de Marguerye émergent-elles dans une période où l'on serait passé d'une vision positive du modéré à une méfiance à

l'égard du possible contre-révolutionnaire masqué. Pour contredire une telle suspicion, Marguerye fournit des certificats de résidence et de civisme, jalonnant ses déplacements en France de 1792 à 1794, et qui constituent en sus des lettres une ressource documentaire originale.

3 Si cette étude est en partie consacrée aux opinions de Marguerye durant l'année 1791, le reste de son existence est connu par ses actes, et révèlerait plutôt ses intérêts. Ces deux termes étant au cœur d'un ouvrage de Joseph Fiévée, "clé de lecture possible à qui veut comprendre les volte-face de toutes les périodes, révolutionnaire, directoriale, consulaire, impériale sans oublier celle de la Restauration ${ }^{5}$ ", nous espérons que leur utilisation participera à éclairer un parcours ne manquant pas de zones d'ombre.

4 Ainsi cette étude de cas se situe-t-elle en relation avec une réflexion plus générale sur la modération et les modérés contemporains de Marguerye ${ }^{6}$. Comment ce dernier a-t-il géré ses intérêts de part et d'autre de cette année charnière que fut pour lui 1791 ? 
Comment, accompagné pour le meilleur et pour le pire de son passé, les a-t-il alors et par la suite conciliés avec les opinions manifestées dans ses lettres de cette année-là ?

1758-1791 : les belles années de Charles de MargueryeUne réussite sociale à la fin de l'Ancien RégimeEntrer dans une carrière

5 Les Marguerye "de Colleville ${ }^{7}$ ", depuis au moins la deuxième moitié du XVIIe siècle jusques et y compris le père et l'oncle de Charles,

semblent s'être conformés à un modèle provincial de noblesse militaire ${ }^{8}$ : carrières en général assez courtes pour les aînés, enclins à se retirer après leur mariage, le célibat des cadets étant de rigueur.

6 À la génération suivante, l'aîné, Bon-Henri-Marie, né à Valognes en $1754^{9}$, ne servit que quelques années dans le régiment Commissaire général cavalerie, sans quitter le grade de sous-lieutenant ${ }^{10}$.

7 Quant au puîné, Charles-Léonor-Louis, baptisé à Valognes le 18 juin 1758, il fit certes de modestes débuts (1er mai 1773) comme lieutenant au régiment provincial de Caen. Mais dès janvier 1775, dans sa dix-septième année, il fut agréé pour une des six charges d'exempt des gardes du corps du comte d'Artois, ce qui lui donnera rang de capitaine de cavalerie ${ }^{11}$.

8 Pourquoi lui et non son frère ? «Ce qui compte, c'est moins le statut d'aîné que les stratégies de fortune des familles et les occasions qui les favorisent ${ }^{12}$ ». La position de l'avocat parisien Élie de Beaumont, natif lui aussi du Cotentin, a pu jouer : ce juriste fut, dès la création du conseil du comte d'Artois en 1773, l'un des trois intendants de ses maisons et finances, jusqu'à sa mort en $1786{ }^{13}$.

9 Nommé en janvier 1775, et non à la création des deux compagnies de gardes deux ans plus tôt, Charles de Marguerye a dû acheter sa charge. À défaut d'avoir retrouvé le contrat d'acquisition, se référer à des cas comparables ${ }^{14}$ permet d'envisager un prix de 60 à 75000 livres. Mise de fonds importante pour une famille de noblesse moyenne, dont on peut évaluer le revenu annuel à moins de 16000 livres ${ }^{15}$; mais le 7 avril 1781, Charles fut ainsi le premier de la famille à monter dans les carrosses du roi, qualifié de comte pour la circonstance, après avoir fait ses preuves devant Chérin, généalogiste des ordres du roi ${ }^{16}$. Il avait bientôt vingt-trois ans.

Gérer des intérêts familiaux

Le frère de Charles s'est marié l'année suivante à l'âge de vingt-huit ans, et il semble bien que son alliance, d'un autre niveau que celles de ses ancêtres ${ }^{17}$, doive beaucoup à la présence du cadet à la cour. Par contrat du 29 décembre 1782 signé du roi et de la famille royale, il épousa une fille du défunt marquis de Séran d'Audrieu, demoiselle de Saint-Cyr dont la mère et la sœur étaient dames d'honneur de la duchesse de Bourbon ${ }^{18}$.

11 Le contrat stipulait une dot de 110000 livres, dont 105000 en avancement d'hoirie de la mère de la future, réservée à sa succession et à d'autres éventuelles, Bon-Henri-Marie l'étant à celle de ses parents, tout en bénéficiant dès le mariage de la jouissance de certains revenus fonciers ${ }^{19}$.

12 Moins de deux ans après son aîné, les 6 et 8 juin 1784, Charles de Marguerye, atteignant ses vingt-six ans, signait lui aussi (avec de nouveau l'agrément du roi et de sa famille) un contrat de mariage ${ }^{20}$. Union moins prestigieuse : la jeune Dupérier Dumouriez était fille d'un défunt trésorier de France au bureau des finances de la généralité de Montauban. 

résidait avec sa mère, qui la réservait dans sa succession. Elle apportait en dot sa part dans l'héritage paternel, comportant un legs de 150000 livres, quasiment 3000 livres de rentes viagères sur sa tête, et des espérances certaines. Quant au futur, il était réservé aux successions de ses parents, qui lui assuraient une pension de 1200 livres, à quoi s'ajoutaient 3000 livres

d'effets mobiliers. Même compte tenu de sa charge de sous-lieutenant des gardes d'Artois, produisant 3000 livres, et d'une pension royale de 800 livres ${ }^{21}$, on peut dire que Charles faisait un bon mariage. D'autant que si un douaire de 3000 livres était prévu, il était en fait assuré par le comte d'Artois qui, par décision de son conseil du 9 mai 1784, promettait 3000 livres de rentes viagères à l'épousée du jour du décès de son mari ${ }^{22}$.

à Colleville-sur-mer ${ }^{24}$. Leur aïeul paternel fut inhumé à Valognes le 16 novembre $1787^{25}$, où le partage sous seing privé de sa

succession fut effectué le 6 avril $1788^{26}$. L'aîné héritait de l'essentiel des biens cotentinais, Charles obtenant 12000 livres de biens immobiliers à Valognes et des biens seigneuriaux, notamment à Colleville-sur-mer.

Une tentative d'adaptation au nouveau régimeD'un rôle politique aux éclairages d'une correspondance

15 Sans doute présent auprès du comte d'Artois, notre noble possédant fief fut représenté aux assemblées de son ordre à Coutances et à Caen en mars $1789^{27}$. Cependant, il fut le 7 février 1790 le premier maire élu de sa commune, prêtant serment de maintenir de tout son pouvoir la constitution du royaume, d'être fidèle à la nation, à la loi et au roi ${ }^{28}$. L'édile n'évoquant quasiment pas l'exercice de son mandat dans les lettres adressées à Blondel de Nouainville en 1791, nous ne nous étendrons pas sur celui-ci ${ }^{29}$. En revanche, il y fait part d'opinions politiques à l'échelle du royaume.

16 Le 27 mars 1791, il présentait à Blondel une source d'information privilégiée : «L'amy des patriotes, également éloigné des aristocrates comme des démagogues, mais amy de la constitution dans ses bazes. Jusqu'à présent, il rend tout ce que je pense. Il a fait beaucoup d'impression, même sur ceux qui désirent la contre révolution " ${ }^{30}$.

17 En ce début de printemps 1791, Marguerye revenait chez lui après une absence de six semaines, ayant passé quinze jours à Paris, et le reste en route ou en Touraine. Avant d'évoquer une vie parisienne qu'il jugeait peu changée par la Révolution, il parle de la «fermentation» due au départ des tantes de Louis XVI pour Rome le 19 février ${ }^{31}$. «Mais j'ai remarqué que l'agitation ne passait pas la dernière classe d'hommes »: formulation à connotation passablement méprisante, pourtant banale à l'époque.

Depuis six semaines, ajoute-t-il, «l'assemblée nationale a rendu beaucoup de décrets qui donnent de la force au roy et aux ministres pour le gouvernement. Toutes les discutions se sont passées sans troubles; le peuple ne s'en est point meslé! Et je vois avec une satisfaction indicible que l'ordre renait $"$.

19 C'est pourtant avec la même détermination qu'il clôt sa missive, exhortant son ami à ne jamais oublier "que nous avons une patrie avant d'avoir un Roy", et à comprendre «que toute contre révolution étant phisiquement et encore plus moralement impossible, il faut se rallier à la constitution pour avoir l'ordre et la perfectionner ».

Annales historiques de la Révolution française, 343 | janvier-mars 2006 
20 Il y aurait quelque anachronisme à inférer que Marguerye n'était ni à gauche, ni à l'extrême-droite ${ }^{32}$. Mieux vaut le situer relativement à un parti de l'assemblée nationale.

21 Il exprime dans sa lettre du 20 janvier, et surtout dans celle du

27 mars, de la défiance vis-à-vis du triumvirat, nommément les Lameth et Barnave, " connus pour des factieux », le crédit du club des Jacobins se perdant, selon lui, en ce début de printemps 1791, sans s'expliquer davantage. Ses propos en faveur de la constitution justifient pourtant qu'on le rapproche du groupe des constitutionnels.

Parmi eux, les monarchiens défendaient comme Marguerye la force de l'exécutif et de l'ordre public. En effet, s'il fallait pour lui « se rallier à la constitution ", c'était " pour avoir l'ordre et la perfectionner ", à n'en pas douter à l'avantage du roi et des ministres ${ }^{33}$. Mais là encore, on ne peut lui faire dire plus qu'il n'a écrit.

23 Nous limitant donc à l'appellation de constitutionnel, nous allons observer ses réactions face aux principaux événements de l'année 1791 à travers sa correspondance. Un constitutionnel, les problèmes religieux et la fuite du roi

24 Le 10 mars 1791, le pape avait rejeté la Constitution civile du clergé. Marguerye considérait que la constitution résisterait à ce choc : « Il est des provinces entières, quoi qu'en disent les journaux aristocrates, où le serment est généralement presté ; et si c'est un tort morale, c'est au moins un bien dans ce moment. La forme du culte n'importe généralement pas assez pour qu'il en résulte une guerre de religion », écrivait-il à la fin du même mois.

Voilà un élément essentiel de sa pensée politique à ce moment-là : la paix civile avant tout. Est-il osé de mettre en parallèle ce jugement sur la religion et sa forme, avec ce qu'il a écrit sur la patrie et le roi ? Si la forme de la religion ne pouvait être la cause d'une guerre de religion, le roi, tout au moins la forme de son pouvoir, ne pouvait causer une guerre civile. Il semblait pressentir que, de toute façon trop faible pour réussir, une contre-révolution ne pourrait se faire que contre la patrie, ce qu'il n'acceptait pas, même au nom du roi et de Dieu.

La lettre du 24 juillet conseille implicitement à son destinataire la prestation du serment du 22 juin précédent, qui ne faisait plus référence au roi coupable d'avoir tenté de fuir : «Vous connaissez mes opinions. Elles ne varient pas. Je suis plus convaincu que jamais que la France est perdue si tout le monde ne se rallient pas à la constitution ; mais je respecte les

opinions de tous les partis, et surtout celles de gens aussi estimables que vous. Bien sûr que, si vous faites une déclaration de deffendre nos nouvelles loix, vous ne vous armerez jamais contre elles, quelque soit votre façon de penser. Vous ferez alors l'action la plus sublime : celle de sacrifier votre

opinion particulière à la tranquilité public ». Varennes n'a pas fait changer le cap de Marguerye.

Le maire de Colleville et son conseil saluèrent le 2 octobre la récente acceptation de la constitution par le roi ${ }^{34}$. «Je crois que son unique parti est d'épouser la constitution, de régner par elle et de faire changer peu à peu ce qu'elle peut avoir de faible dans les moyens d'exécution ", avait confié Marguerye dans sa lettre à Blondel du 21 septembre. Constance d'opinion de celui qui a pu critiquer des politiciens (Mirabeau, le 20 janvier), voire les Français, « impatients du joug sans savoir être libres » (10 juin), mais jamais la monarchie constitutionnelle. 

retraite. Par décision du 18 décembre suivant, il obtint un brevet de lieutenant-colonel du 7e régiment de cavalerie, ex-Royal-Etranger ${ }^{42}$. Il répugnait à la plupart des anciens membres des maisons 
militaires des princes de solliciter des nominations « constitutionnelles ${ }^{43}$. Marguerye ne s'arrêta pas à ces préventions, en accord avec les opinions exprimées dans ses lettres.

La charnière

Sa bonne volonté ne put le prémunir d'un conflit local qui dura du début de l'automne 1791 à la fin du printemps $1792{ }^{44}$. Il procède d'un démêlé au château de Colleville entre un gagne-denier de la commune et le propriétaire des lieux. Peu après, soutenu par le curé ${ }^{45}$, le journalier signa une requête adressée à la Société des Amis de la constitution de Caen. Le châtelain était implicitement suspecté de contre-révolution. Le renouvellement de l'assemblée municipale de Colleville, prévu le 20 novembre, fut reporté, le maire, entre autres, étant absent. C'est pourtant en son absence qu'il fut réélu à l'unanimité le 4 décembre ${ }^{46}$.

La requête ${ }^{47}$ fut appuyée le 3 décembre par les Jacobins caennais auprès de l'administration départementale. Celle-ci chargea le procureur-syndic du district de Bayeux d'éclaircir l'affaire. Un mois plus tard, la justice de paix transmettait les résultats de son enquête, à laquelle n'avait pas participé un Marguerye toujours absent. Éloignement sans doute dicté par la prudence, malgré des témoignages dans l'ensemble favorables.

L'affaire n'est close que le 16 mars 1792 en directoire à Caen par la disculpation du maire, le curé étant plus ou moins suspecté d'avoir manipulé le journalier ${ }^{48}$. Ces inimitiés auront sans doute davantage le champ libre dans la période troublée qui s'annonce.

Les pérégrinations d'un " centriste perdu » ${ }^{49}$

Le 10 avril 1792, dix jours avant la déclaration de guerre «au roi de Bohême et de Hongrie », le registre des délibérations de Colleville prend acte de la démission du maire dans une lettre datée de Paris du 3 février précédent, faisant état de son absence et de sa rentrée dans un service actif de lieutenant-colonel ${ }^{50}$.

Précisons que dans sa lettre du 21 septembre 1791, Marguerye avait écrit à Blondel que si la guerre avait lieu, il faudrait bien y prendre part, mais seulement si l'ordre était un peu rétabli dans l'armée. Hypothèque à ses yeux levée six mois plus tard ? À moins que les événements locaux survenus entre temps n'aient joué dans sa décision.

C'est à partir d'avril 1792 qu'ont été produits les certificats que nous avons réunis dans un tableau ${ }^{51}$. L'authenticité et la date réelle d'établissement des documents enregistrés (généralement des copies certifiées conformes) posent de redoutables problèmes, nous laissant dans l'incertitude. Nous pouvons néanmoins essayer de répondre à deux questions : pourquoi ont-ils été produits? Avec quels résultats?

41 La tentative de réponse sera suivie de l'étude de la période allant de la fin 1794 à la fin 1799, cinq années pour lesquelles nous sommes nettement moins documentés sur le parcours que nous essayons de suivre.

Les certificats de Marguerye enregistrés à Colleville-sur-Mer de 1792 à $1794{ }^{52}$

\begin{tabular}{|l|l|l|l|l|}
\hline $\begin{array}{l}\text { Nature et } \\
\text { dates }\end{array}$ & Lieux de production & Auteurs & $\begin{array}{l}\text { Enregistrement } \\
\text { à Colleville }\end{array}$ & $\begin{array}{l}\text { Informations } \\
\text { sélectionnées }\end{array}$ \\
\hline
\end{tabular}




\begin{tabular}{|c|c|c|c|c|c|}
\hline 1) & $\begin{array}{l}\text { résidence } \\
24 \text { avril } 1792\end{array}$ & Commercy & $\begin{array}{l}\text { municipalité, } \\
\text { visa district }\end{array}$ & 30 avril 1792 & $\begin{array}{lr}\text { garnison } & \text { du7e } \\
\text { régiment } & \text { de } \\
\text { cavalerie } & \end{array}$ \\
\hline 2) & $\begin{array}{l}\text { militaire } \\
\text { 14-15-16août } \\
1792\end{array}$ & Sedan & $\begin{array}{l}\text { Conseil } \\
\text { d'administration } \\
\text { 7e rgta cavalerie } \\
\text { et municipalité ; } \\
\text { visa du district }\end{array}$ & 22 mars 1793 & $\begin{array}{l}\text { au corps depuis } \\
\text { janvier (Sedan: } \\
\text { campement } \\
\text { du7e régiment } \\
\text { de cavalerie) }\end{array}$ \\
\hline 3) & $\begin{array}{l}\text { résidence } \\
20 \text { oct. } 1792\end{array}$ & $\begin{array}{l}\text { St-Marcel-les- } \\
\text { Clavy }^{53} \text { (district de } \\
\text { Charleville) }\end{array}$ & $\begin{array}{l}\text { municipalité, } \\
\text { visa district }\end{array}$ & 23 mars 1793 & $\begin{array}{l}\text { depuis le } 20 \\
\text { août } 1792 ; \mathrm{y} \mathrm{a} \\
\text { prêté serment } \\
\text { selon formule } \\
\text { du } 10 \text { août }{ }^{54}\end{array}$ \\
\hline 4) & $\begin{array}{l}\text { résidence } \\
5 \text { déc. } 1792\end{array}$ & Tours & $\begin{array}{l}\text { municipalité et } \\
\text { district }\end{array}$ & 25 mars 1793 & $\begin{array}{l}\text { depuis le } 25 \text { nov. } \\
1792\end{array}$ \\
\hline 5) & $\begin{array}{l}\text { résidence } \\
16 \text { février } \\
1793\end{array}$ & $\begin{array}{l}\text { St-James } \\
\text { (département de la } \\
\text { Manche, district } \\
\text { d'Avranches) }\end{array}$ & $\begin{array}{lr}\text { municipalité } & \\
\text { visa } & d u \\
\text { directoire } & d u \\
\text { département } & \text { à } \\
\text { Coutances } & \end{array}$ & 25 mars 1793 & $\begin{array}{ll}\text { à } & \text { Villiers } \\
\text { (canton de St- } \\
\text { James) depuis le } \\
12 \text { déc. } 1792^{55}\end{array}$ \\
\hline 6) & $\begin{array}{l}\text { civisme } \\
10 \text { avril } 1793\end{array}$ & Colleville-sur-mer & municipalité & & \begin{tabular}{|lr} 
"pendant trois \\
ans maire \\
ayant d...] \\
dans tous les \\
temps des \\
preuves de \\
patriotisme"
\end{tabular} \\
\hline 7) & $\begin{array}{l}\text { résidence } \\
22 \text { juin } 1793\end{array}$ & Rouen & municipalité & 30 sept. 1793 & $\begin{array}{l}\text { depuis le } 5 \text { mars } \\
1793\end{array}$ \\
\hline 8) & $\begin{array}{l}\text { militaire } \\
3 \text { sept. } 1793\end{array}$ & Vitry ${ }^{56}$ & $\begin{array}{l}\text { Conseil } \\
\text { d'administration } \\
\text { du } 7 \text { e rgt de } \\
\text { cavalerie }\end{array}$ & 25 octobre 1793 & $\begin{array}{l}\text { présent de mars } \\
1792 \mathrm{au} 17 \text { août } \\
1792 \\
\text { (démission) }^{57}\end{array}$ \\
\hline
\end{tabular}




\begin{tabular}{|c|c|c|c|c|c|}
\hline 9) & $\begin{array}{l}\text { civisme } \\
\text { (couple } \\
\text { Marguerye) } \\
20 \text { janvier } \\
1794\end{array}$ & Colleville-sur-mer & municipalité & & $\begin{array}{l}\text { importance de } \\
\text { leurs dons } \\
\text { patriotiques au } \\
\text { regard de leur } \\
\text { fortune; départ } \\
\text { pour Rouen } \\
\text { regretté ; } \\
\text { justifié en ce qui } \\
\text { concerne } \\
\text { l'épouse par son } \\
\text { état de santé }\end{array}$ \\
\hline 10) & $\begin{array}{l}\text { résidence } \\
14 \text { avril } 1794\end{array}$ & Rouen & $\begin{array}{l}\text { municipalité } \\
\text { (« commune } \\
\text { révolutionnaire ») }\end{array}$ & \begin{tabular}{|lr}
6 & sept. \\
1794 (enregistré \\
à Caen dès le 24 \\
avril 1794)
\end{tabular} & $\begin{array}{l}5 \text { mars au } 2 \\
\text { sept. et depuis } \\
\text { le18 octobre } \\
1793 \text { « vivant de } \\
\text { son revenu» }\end{array}$ \\
\hline 11) & $\begin{array}{l}\text { civisme } \\
12 \text { nov. } 1794\end{array}$ & $\begin{array}{l}\text { [St] «Meslain du } \\
\text { Bosc » (département } \\
\text { de l'Eure, district } \\
\text { deLouviers) }\end{array}$ & municipalité & 28 déc. 1794 & $\begin{array}{l}\text { négociant; } \\
\text { "s'est toujours } \\
\text { comporté en } \\
\text { bon citoyen et } \\
\text { vray } \\
\text { républicain " }\end{array}$ \\
\hline 12) & $\begin{array}{l}\text { résidence } \\
28 \text { nov. } 1794\end{array}$ & Colleville-sur-mer & municipalité & & $\begin{array}{l}\text { depuis le } 3 \\
\text { septembre 1794, } \\
\text { "vivant de son } \\
\text { revenu " }\end{array}$ \\
\hline
\end{tabular}

De 1792 à la fin 1794 incluait plusieurs Marguerye (ou Marguerie), dont l'un sans prénom ${ }^{62}$, et le 6 novembre 1792, il figurait comme possessionné à Valognes sur une liste d'émigrés ayant des biens 
dans le département de la Manche ${ }^{63}$. Dans ces conditions, même si la société populaire créée dans son village le 22 juillet 1792 cessa de se réunir dès le 23 septembre de la même année ${ }^{64}$, l'ancien seigneur et maire pouvait craindre le réveil de malveillances qui eussent compromis sa sûreté. Raison de plus pour convaincre les autorités qu'il n'était pas un émigré.

Constatant que le certificat de Sedan de la mi-août $1792\left(\mathrm{n}^{\circ} 2\right.$ du tableau) ne fut connu à Colleville qu'au début du printemps 1793, nous comprenons pourquoi, sur réquisition du district de Bayeux, la petite municipalité déclara le 21 décembre 1792 ignorer ce qu'était devenu son ancien maire, se contentant d'établir un état de ses biens dans la commune ${ }^{65}$; ce qui n'augurait rien de bon pour le propriétaire.

Le 10 avril 1793, un certificat de civisme de Colleville ( $n^{\circ} 6$ du tableau) affirmait que l'intéressé n'était ni père, ni fils, ni frère d'émigrés. Et si le 28 novembre $1794\left(\mathrm{n}^{\circ} 12\right)$ Pierre Josse ${ }^{66}$ pouvait écrire que Marguerye n'était pas sur la liste des émigrés et ses biens pas séquestrés, c'est peut-être parce qu'il avait déjà pu lire une telle formulation dans le certificat rouennais du 14 avril précédent $\left(n^{\circ} 10\right)$ faisant référence à une attestation du directoire de la Seine-inférieure du 18 mars; mais c'est plus probablement parce que son ancien maire lui fit alors part de deux documents essentiels, mais non enregistrés à Colleville: un certificat obtenu du directoire du Calvados le 23 septembre 1793 qui s'exprimait déjà en ces termes favorables à son égard, et surtout un arrêté du directoire de la commission administrative de la Manche du 15 décembre suivant le comprenant dans la radiation provisoire de 152 personnes de la liste des émigrés de ce département ${ }^{67}$.

Marguerye n'était pourtant pas alors rentré à Colleville, nonobstant un décret de la Convention du début du mois de septembre 1793 ordonnant à certains suspects (dont les militaires démissionnaires et les anciens membres des ci-devant maisons militaires des frères de Louis XVI) de se retirer dans leurs municipalités dans les vingt-quatre heures, munis d'un passeport du ministre de la Guerre, sous peine de dix ans de fers ${ }^{68}$. Ce qui peut amener à s'interroger sur la portée réelle de telles injonctions.

Notre homme n'a donc pas eu besoin d'attendre la réaction thermidorienne pour voir sa situation commencer à s'améliorer. L'an II lui fut même plutôt bénéfique, ce qui n'est peut-être pas sans rapport avec «la modération même de la Terreur de l'an II en Normandie ${ }^{69}$ ». Qu'en a-t-il été par la suite?

De la fin 1794 à la fin 1799

50 Les délibérations municipales de Colleville-sur-mer restent quasiment muettes sur le châtelain du lieu de l'automne 1794 à la Restauration ${ }^{70}$. Il ne s'y serait pas réinstallé durablement puisqu'en juin 1795, venu à Paris contracter une obligation conjointe avec son frère ${ }^{71}$, il se dit demeurant à Rouen et négociant ${ }^{72}$. Pour cette période qui va de la fin 1794 à la fin 1799, nous ne disposons donc plus de certificats, encore moins de lettres, seulement de quelques actes notariés.

51 Dès l'été 1797, Charles de Marguerye était installé dans la capitale, près de la place Vendôme ${ }^{73}$, domicilié en janvier 1799 rue du faubourg Saint-Honoré, se qualifiant de citoyen français ${ }^{74}$. Ainsi, nous sommes peu renseignés sur ses activités quand conventionnels, puis directeurs, maintinrent une politique discriminatoire à l'égard des ci-devant nobles ${ }^{75}$.

52 Jusqu'à Brumaire, le Directoire s'efforça en effet de les écarter des radiations des listes, bien qu'à partir de floréal an VII (avril-mai 1799) une majorité modérée eût imposé ses 
vues à l'égard des émigrés. Le 17 messidor an VII (5 juillet 1799), une loi vint régler l'ordre des radiations de ces listes d'émigrés ${ }^{76}$; et c'est en conséquence de celle-ci que Marguerye produisit au Ministère de la Police générale plusieurs certificats.

Le rapport du sous-chef de la division des émigrés, qui rappelait que Rouen était une fabrique connue de faux certificats, ne le ménageait pas: élevé en grade en 1791, époque où la cour favorisait ceux qui lui étaient le plus dévoués, il a quitté le service après le 10 Août, quand la plupart des chefs de corps ont émigré ; donc il a dû se rendre à Coblence comme les

autres; s'il est rentré, cela signifie-t-il qu'il n'a pas été un ennemi de l'intérieur ${ }^{77}$ ? D'ailleurs, d'autres Marguerye ont émigré. Certains ont même été des contrerévolutionnaires actifs... ${ }^{78}$

Le 1er août 1799 , les pièces sont donc envoyées pour vérification au commissaire du Directoire près l'administration départementale de la Manche. Une lettre à lui adressée de Saint-James le 14 août signale qu'un grand nombre des témoins suscités en 1793 «sont des hommes de qui on obtiendrait tout contre le gouvernement pour de l'argent ${ }^{79}$.

55 Encore le rapport n'en rajoute-t-il pas sur le séjour de quelques mois à Villiers près Saint-James chez le citoyen Tuffin (certificat $n^{\circ} 5$ du tableau), ce personnage étant non seulement cousin de Marguerye, mais aussi de La Rouërie. Soupçonné d'avoir eu part à la célèbre coalition bretonne depuis mars 1792, il fut décrété d'arrestation en juin 1793, et mourut sous les coups des Bleus en mai $1794^{80}$. Que notre Normand avisé se soit réclamé d'une telle domiciliation peut laisser perplexe. Que des autorités en quête d'éléments à charge aient laissé passer celui-là encore plus.

Le 3 octobre 1799, le commissaire susdit communique au ministre de la Police l'extrait de la radiation provisoire du 15 décembre 1793. Le 13 octobre 1799, le rapport du 1er bureau de la division des émigrés n'en conclut pas moins qu'il faut demander des renseignements au ministre de la Guerre et aux départements de la Manche et de la Seine-inférieure, et juge que l'intéressé ne peut jouir de la faveur de la loi du 17 messidor an VII ${ }^{81}$.

Improbables reclassements (1803-1840)Ralliement à Bonaparte

On ne sait si Charles de Marguerye a bénéficié des mesures d'amnistie consulaires. Ce n'est cependant pas son ralliement qui étonne, sinon par sa matérialisation dans un domaine auquel rien ne semblait le prédisposer : les finances publiques; mais plutôt l'intérêt que le régime avait à le rallier, l'« habileté du gouvernement consulaire [tenant] précisément dans cette distribution de faveurs qui émousse la résistance des plus irréductibles ${ }^{82} »$. On ne peut que supposer une puissante intervention en sa faveur.

Le 8 décembre 1803, il fut en effet nommé receveur particulier de l'arrondissement de Roanne ${ }^{83}$, mais surtout promu par décret impérial du 23 mai 1806 receveur général du département de l'Indre, choisi par Napoléon lui-même au détriment de deux candidats, dont le ministre des Finances ${ }^{84}$ avançait les noms, et de vingt-trois autres, déjà connus ou recommandés, dont il joignait une liste, où Marguerye ne figurait pas ${ }^{85}$.

Pierre-François Pinaud a identifié neuf nobles d'Ancien Régime parmi les receveurs généraux en poste entre 1800 et 1815, Marguerye lui ayant échappé ${ }^{86}$. Sur cette base, nous avons relevé cinq anoblis du XVIIIe siècle, dont trois de familles déjà liées à la finance ${ }^{87}$. Parmi les quatre receveurs 
de noblesse antérieure, un seul peut être mis sur le même plan que Marguerye. Nous sommes évidemment loin des quatorze lieutenants et sous-lieutenants des gardes d'Artois à la veille de la Révolution, dont huit appartenaient à des familles de vieille noblesse, trois d'entre-elles présentées à la cour ${ }^{88}$.

Le nouveau receveur fut en relation avec de nombreux manieurs d'argent pour l'escompte d'effets de commerce, cherchant ainsi à faire fructifier l'argent de l'État à son profit avant de le verser ${ }^{89}$. Pratique courante, où chacun trouvait son compte, à commencer par les receveurs qui complétaient ainsi largement leur traitement annuel de 6000 francs ; mais en cas de faillite, l'État retrouvait sa rigueur ${ }^{90}$.

61 Or, le 23 juillet 1810, une inspection révéla un déficit qu'était loin de compenser une caution dépassant pourtant 130000 francs. Dès le lendemain, le fautif était suspendu. S'ensuivit la mise sous scellés de ses biens sur place et sa mise en faillite en tant que banquier par le tribunal de commerce de Châteauroux ${ }^{91}$.

Des mauvaises affaires de notre homme, il ressort que le déficit excédait 350000 francs, « en y comprenant les effets revenus à protêt, et ceux que le comptable faisait apparaître par double emploi dans son portefeuille, ou représentait comme étant à recouvrer, quoiqu'il en eût reçu le montant ${ }^{92}$.

63 Le tribunal civil de Châteauroux ordonna en faveur d'un Trésor public prioritaire sur les particuliers la levée des scellés, la vente des biens meubles et effets mobiliers, et le recouvrement des créances et revenus de Marguerye ${ }^{93}$. Celui-ci est révoqué par décret daté de Saint-Cloud le 31 août $1810^{94}$.

64 Pourtant, dès le printemps 1811, estimant la situation de Marguerye plus satisfaisante et le paiement de ce qu'il doit garanti, le ministre du Trésor public l'autorise à revenir à Châteauroux (dont notre homme s'était prudemment éloigné durant les temps difficiles) pour accélérer le règlement de ses affaires. Le 29 septembre 1810, dans une lettre au préfet de l'Indre, Mollien avait d'ailleurs été très clair: "Le sieur de Marguerye ne peut trop s'empresser de fournir des sûretés au Trésor, s'il ne peut immédiatement rembourser son déficit. Ce n'est que par sa prompte et entière libération envers le Trésor public qu'il peut espérer quelques droits à

l'indulgence de Sa Majesté ». Le dossier n'était toujours pas clos quinze ans plus tard ${ }^{95}$.

Avant d'en venir aux nouveaux espoirs qu'a pu susciter chez Marguerye la chute de l'Empire, interrogeons-nous sur l'attitude des autres anciens lieutenants et souslieutenants des gardes d'Artois à l'égard du régime bonapartiste. Un sous-lieutenant, rentré en France en 1800, décéda à Versailles onze ans plus tard; un autre, Capdeville, également revenu

d'émigration, aurait recherché en 1808 le commandement d'une garde d'honneur à cheval lorsque l'Empereur vint à Pau, peccadille qu'il aurait tue ou niée quelques années après ${ }^{96}$. Quant au lieutenant Mousin, il sut dès son retour gagner les bonnes grâces de Kellermann, et saisit toutes les occasions pour demander au ministre de la Guerre son retour à un service militaire actif, "en sacrifiant mes jours à mon souverain ». Il fut constamment éconduit, mais obtint pour ses deux fils une place dans un lycée, puis à l'École militaire de Fontainebleau, et put ensuite faire état de leurs services comme officiers ${ }^{97}$.

Ces quelques exemples montrent que, même au sein d'un corps privilégié, les ralliements n'étaient pas rares. L'originalité de Marguerye réside plutôt dans la nature 
de la place qu'il remplit, et dans les circonstances de sa perte. Était-ce là condition favorable lors du retour des Bourbons ${ }^{98}$ ?

Ralliement aux Bourbons

67 Le 15 juillet 1814, Charles de Marguerye retrouva son emploi de sous-lieutenant des gardes du comte d'Artois devenu "Monsieur». Il fut promu second-lieutenant le 24 septembre suivant. Entre-temps, il avait obtenu la croix de Saint-Louis, et fut reçu dans cet ordre par le duc de Berry. Dans son mémoire de demande le 15 juin précédent, il tentait de légitimer sa traversée de la Révolution, en s'attribuant quelques services pour la cause des princes: émigré en 1792, il aurait fait campagne comme simple chasseur volontaire dans les chasseurs russes de l'armée des princes ${ }^{99}$. Il n'aurait cessé d'être aux avant-postes, notamment à celui qui fut attaqué près de Châlons, où plusieurs de ses camarades auraient été tués ou pris. «Ayant perdu son cheval avec son porte-manteau qui contenait toutes ses ressources, il rentra alors en France lors de la désorganisation à Arlon " ${ }^{100}$, où, à la mi-octobre 1792, une bonne partie des émigrés, avec l'autorisation des princes, quittèrent l'armée dans les pires conditions matérielles et morales. Marguerye s'intègre donc dans ce reflux comme pour s'y noyer ${ }^{101}$, d'autant qu'il ne peut faire état d'aucun service postérieur ${ }^{102}$.

Le 19 janvier 1815, il joignait à la croix de Saint-Louis celle de chevalier de la Légion d'honneur, et le 12 février, il était fait 1er lieutenant des gardes de Monsieur, avec rang de colonel, selon l'ordonnance qui avait rétabli ces compagnies de gardes.

Quand viennent les Cent-Jours, il suit Louis XVIII, ce qui était sans doute agir selon son cœur, mais aussi selon son intérêt. Qu'espérer d'un nouveau ralliement à Napoléon ? Et puis, 1815 permettait de rejouer 1792. Il n'eut en tout cas rien à regretter : commandant sous le duc de Berry

l'escadron des gardes de Monsieur jusqu'à la rentrée du roi, il fut promu à Gand le 14 juin officier dans l'ordre de la Légion d'honneur. Et au retour, il négocia sa retraite : par décision royale du 22 novembre, il obtint le grade honorifique de maréchal de camp, et par une autre du 7 avril 1816 une pension de 4500 francs, dont il se proposait d'aller jouir à Colleville ${ }^{103}$.

70 Quant aux autres lieutenants et sous-lieutenants des gardes d'autrefois, ils furent gratifiés des grades honorifiques pour retraites de maréchal de camp ou de lieutenant général, qu'ils aient ou non repris du service en 1814, suivi ou pas le roi à Gand. Néanmoins, des deux qui furent distingués par le titre de commandeur de Saint-Louis, le vicomte de Gouvello avait, comme Marguerye, additionné ces deux preuves de fidélité. Mais Gouvello serait resté en Angleterre jusqu'en $1814^{104}$. Pour un officier demeuré en France, un temps au service de Bonaparte, Marguerye fut assez bien loti.

La retraite

71 Revenu habiter Colleville, il y retrouva le 15 mai 1817 son fauteuil de maire, remplaçant Michel Le Verdier, mis en place par Napoléon. Le 23 février 1821, il prit acte de l'impossibilité pour la commune de participer à l'acquisition du domaine de Chambord en faveur de "l'enfant du miracle ", invitant ceux qui le pouvaient à faire comme lui une offre individuelle. Le 30 septembre suivant, âgé de soixante-trois ans, il démissionne, pour être remplacé par son fils ${ }^{105}$.

Durant cette période, Marguerye faisait partie du collège électoral d'arrondissement, payant une contribution comprise entre 900 et 950 francs ${ }^{106}$. S'il en disparaît par la suite, c'est en raison de la vente qu'il fit à son fils, le 19 août 1822, de sa terre de Colleville, à charge d'en payer les impositions. Le prix de 69000 francs n'était pas versé, 
mais délégué à

l'épouse du vendeur pour partie de sa créance sur son mari, son fils s'engageant à lui en verser la rente sur le pied de $5 \%$ l'an ${ }^{107}$.

73 Le père réapparaît de façon suivie dans ce collège électoral à la charnière de la décennie suivante, en qualité d'officier en retraite ayant au moins 1200 francs de pension ${ }^{108}$. La loi électorale de 1831 exigeait un demi cens de 100 francs pour exercer cette capacité, et les électeurs des monarchies censitaires devaient prêter le serment exigé par ailleurs des fonctionnaires publics ${ }^{109}$. Nous ne pouvons rien affirmer sur l'usage que fit Marguerye de son droit de vote après les Trois glorieuses ${ }^{110}$.

Il est mort à Bayeux le 2 janvier 1840, à l'âge de 81 ans ${ }^{111}$. En raison de la séparation de biens, l'inventaire après décès à Colleville et Bayeux se limite à quelques effets et papiers personnels ${ }^{112}$. L'épouse rejoignit son mari le 11 juillet 1843, à l'âge de 74 ans ${ }^{113}$, la déclaration de mutation après décès indiquant que la défunte percevait un douaire sur la succession de Charles $\mathrm{X}^{114}$, conformément à l'engagement pris par le comte d'Artois dans le contrat de mariage de 1784 .

«Est girouette celui qui, depuis 1789, a prêté serment ou bien a rendu public son attachement "indéfectible" en faveur de deux au moins des régimes qui se sont succédé jusqu'en mars $1815^{115} \%$. Marguerye, comme beaucoup de ses contemporains, correspond à ce critère défini cette année-là par les auteurs d'un Dictionnaire et d'un Almanach des girouettes ${ }^{116}$. Si nous ne l'y avons pas trouvé, c'est sans doute parce qu'il était trop petit personnage. Mais était-ce une « girouette » ?

Il fait partie de ceux qui ont beaucoup prêté serment, en tout cas avant 1830 ; néanmoins, cette docilité peut être regardée, en accord avec ses opinions de 1791, comme une forme d'attachement à une légitimité : celle d'un pouvoir exécutif prééminent, fondé sur une constitution et assurant l'ordre public. Ce qui n'exclut pas un attachement particulier à la première monarchie selon la charte.

Le fils fut moins pressé par la nécessité que le père, et pourtant: maire rallié à la Monarchie de Juillet, quand même remplacé par Michel Le Verdier dès septembre 1830, il resta dans le conseil municipal jusqu'à sa mort en 1855, après moult serments ${ }^{117}$.

Lorsque Charles-Louis est devenu chef de la branche de Colleville en 1840, le patrimoine ${ }^{118}$ et le rang social de celle-ci restaient conséquents. Quant à la réputation laissée par le défunt comte, nous ignorons si elle fut atteinte par certains aspects de son parcours.

79 C'est évidemment surtout à ses démêlés avec le Trésor public que nous pensons. Même si paradoxalement ils ont pu faciliter son ralliement aux Bourbons en 1814, c'est probablement là son principal échec, dans cette première moitié de XIXe siècle où une fonction importante au service de l'État couronnait une carrière ${ }^{119}$.

L'essentiel nous semble pourtant être les opinions du « mitoyen ${ }^{120}$ » de 1791, pouvant peut-être contribuer à "la définition d'une ligne idéologique de la modération ${ }^{121}$ ». Plus modestement, ce qui ne veut pas dire plus facilement, on peut s'interroger sur l'ancrage régional des opinions modérées de Marguerye. Avec beaucoup de nuances quant aux moments, aux endroits, aux personnes, le caractère modéré de la Normandie et de nombreux Normands durant la Révolution a été souligné ${ }^{122}$. L'objet de cette étude pourrait en être un reflet. 


\section{NOTES}

1.Ces lettres sont contenues dans le manuscrit 154 de la Bibliothèque municipale de Cherbourg, base d'une recherche en cours sous la direction de M. Jean-Clément Martin. Afin de ne pas surcharger les notes, et sauf autre indication, les citations seront extraites de cette source, qui comporte également une lettre de 1788, dans laquelle Marguerye s'exprime peu sur les événements de la pré-Révolution, et quatre lettres écrites par son épouse en 1791.

2.À notre connaissance, il avait jusqu'alors uniquement retenu l'attention de Christine Peyrard, « Le maire, le curé, l'évêque et les journaliers de Colleville-sur-mer ou la politique au village en 1791 et $1792 »$, dans Christine LE BOZEC et Eric WAUTERS [dir.], En hommage à Claude Mazauric. Pour la Révolution française, Rouen, 1998, p. 223-228.

3.Concept d'usage courant, mais mal défini.

4.Georges BENREKASSA, « Modération, Modéré, Modérantisme », dans Rolf REICHARDT et Hans-Jürgen LÜSEBRINK, Handbuch politisch-sozialer Grundbegriffe in Frankreich : 1680-1820, Munich, 1996, p. 123-158 (p. 139 pour la citation).

5.Pierre SERNA, La République des girouettes (1789-1815... et au-delà). Une anomalie politique : la France de l'extrême-centre, Paris, 2005, 575 p. , p. 498 ; nous citons ce qu'écrit p. Serna au sujet de l'ouvrage signé par Fiévée en 1809 : Des opinions et des intérêts pendant la Révolution.

6.Ibid.

7.Colleville-sur-mer, berceau de leur branche dans le Bessin.

8.Cf. LA CHESNAYE DES BoIs, Dictionnaire de la noblesse (3e éd., 19 vol. , Paris, 1863-1876),

t. 13, colonnes 230-231. Rappelons que ces mémoires généalogiques étaient généralement fournis par les familles, même si l'auteur ne l'indique pas dans le cas présent.

9.AM, Valognes, registres paroissiaux.

10.Contrôle des officiers : Arch. Guerre, $\mathrm{Yb} 536, \mathrm{f}^{\circ} 57 \mathrm{v}^{\circ}$, et $\mathrm{Yb} 563, \mathrm{f}^{\circ} 49$.

11.Arch. Guerre : dossier personnel, 7Yd 2123 ; maisons des princes, Yd 349 ; contrôle des gardes du corps du comte d'Artois, Yb 77.

12.Emmanuel DE WARESQUIEL, Talleyrand, le prince immobile, Paris, 2003, 799 p. , p. 47.

13.Sandrine BULA, L'apanage du comte d'Artois (1773-1790), Paris, 1993, 260 p. , p. 87, 101-103 et 193.

14.Par exemple Puisaye, qui acquit en 1783 une charge d'exempt des Cent Suisses de la maison du roi pour 70000 livres ; cf. Maurice HUTT, Chouannerie and Counter-Revolution: Puisaye, the Princes and the British Government in the 1790's, Cambridge, 1983, 2 vol. , 630 p. , vol. 1, p. 23-24.

15.Sur la base d'une capitation moyenne de 157 livres de 1769 à 1789 (AD Calvados : C 4619, 4621, 4627 et 4635, capitation des nobles de la généralité de Caen) évaluée au centième du revenu, cf. Guy CHAUSSINAND-NOGARET, La noblesse au XVIIIe siècle. De la féodalité aux Lumières, réédition Paris, 2000, 239 p. , p. 77-78.

16.Arch. Guerre, dossier personnel, 7 Yd 2123 ; et François BLUCHE, « Les honneurs de la cour ", Les cahiers nobles, nos 10-11, 1957, p. 62. La recommandation du chevalier de Crussol, capitaine de compagnie de Marguerye, donc implicitement du comte d'Artois, était ici essentielle. Le généalogiste des ordres du roi reconnaissait à la famille de 
Marguerye une ancienneté de noblesse de cinq siècles : $\mathrm{BN}$, mss, Chérin $130, \mathrm{n}^{\circ} 2675, \mathrm{f}^{\circ}$ 2-11; et AN, MM811, p. 587-595.

17.BN, Chérin 130, fo 23 : minute d'une lettre du 24 octobre 1780 , qui relevait « très peud'alliances de marque » dans la famille Marguerye.

18.Henri WOELMONT DE BRUMAGNE, Notices généalogiques, 8 vol. , Paris, 1923-1925, vol. 5,p. 833-834.

19.AN, Minut. cent., LXXXIII, 613.

20.Ibid., XCIX, 682.

21.Celle-ci récompensait ses « preuves de zèle » à l'égard du comte d'Artois lors du siège de Gibraltar en août-septembre 1782 ; dossier personnel, Arch. Guerre, 7Yd 2123. 22.Arch. nat, Minut. cent., XCIX, 682.

23.Selon son acte de décès du 30 mai 1855, qui lui donne 69 ans un mois; AM Bayeux. 24.AD Calvados, 4 E 2137 : registres paroissiaux de Colleville-sur-mer. Elle eut pour marraine sa grand-tante, épouse Tuffin, qui avait un fils dont nous aurons à reparler. 25.AM Valognes, registres paroissiaux.

26.Pièce jointe à un acte notarié du 19 août 1822 : AN, Minut. cent., XIV, 663.

27.Procès-verbal de l'assemblée générale des trois ordres du grand bailliage de Cotentin, Coutances, 1789, et AD Calvados, 16 B 5 bis, procès verbal (imp., Caen, 1789) de l'assemblée de l'ordre de la noblesse du grand bailliage de Caen (procuration annexée). 28.AD Calvados, 376 Edt 1 : Délibérations municipales de Colleville-sur-mer, 1790-1811.

29.Mme Peyrard a écrit l'essentiel à ce sujet, loc. cit.

30.Dans l'Histoire générale de la presse française, Paris, 1969-1976, 5 t. , t. 1, XV- 633 p. , l'auteur de la partie consacrée à la Révolution et l'Empire, Jacques Godechot, classe L'Amy des patriotes, également titré Le défenseur de la Révolution, dans la " presse contrerévolutionnaire modérée " (p. 467). On ne peut que remarquer le hiatus entre cette qualification et le point de vue d'un lecteur de l'époque.

31.Ce départ fut en effet considéré par certains révolutionnaires comme une émigration de mauvais augure.

32.Marcel GAUCHET, « La droite et la gauche », dans Les lieux de mémoire, sous dir. Pierre NORA, réédition Paris, 1997, 3 t. , t. 2, p. 2533-2601, rappelle (p. 2534) que « c'est aller vite en besogne que de faire surgir la droite et la gauche toutes armées de la matrice révolutionnaire ». Il ajoute (p. 2540) : « En fait de naissance [de la droite et de la gauche], c'est plutôt de faux départ qu'il convient de parler ».

33. Robert GRIFFITHS, Le centre perdu. Malouet et les « monarchiens » dans la Révolution française, Grenoble, 1988, 277 p. , explique, p. 17 : « Pour les monarchiens le pouvoir ultime résidait toujours dans la royauté alors que pour les autres constitutionnels, plus empreints d'idées “démocratiques", c'était l'assemblée qui était prépondérante. » 34.AD Calvados, $376 \mathrm{Edt} 1$.

35.GAUVILLE Louis-Henry-Charles (baron de), Journal du baron de Gauville, député de l'ordre de la noblesse aux états généraux, depuis le 4 mars 1789 jusqu'au 1er juillet 1790, publié pour la première foisd'après le manuscrit autographe [par Édouard de Barthélémy], Paris, 1864, XXVIII-83 p.

36.Si, en octobre 1789, il inclut Malouet ou Virieu dans ce " côté droit » constitué fin août, il se démarque très nettement des "Impartiaux » (dont nommément Virieu) durant le premier semestre 1790 ; Ibid., p. 20, 29, 44, 59. Sur la différenciation entre une droite et un centre (exprimée par la création du Club des Impartiaux en janvier 1790) : R. GRIFFITHS, op. cit., en particulier p. 89-94.

37.Journal, op. cit., p. 53. 
38.Cf. le beau titre du livre de Michel FIGEAC, L'automne des gentilshommes, Paris, 2002, $377 \mathrm{p}$.

39.Selon Louis Hartmann, les maisons militaires des princes avaient été supprimées par un décret du 2 mai 1790, ceux qui les composaient étant susceptibles d'être replacés dans des emplois vacants au choix du roi : Les officiers de l'armée royale et la Révolution, Paris, 1910, IV-540 p. , p. 152. Nous ne sommes pasparvenus à retrouver ce décret. 40.François-Marie-Léon-Robert GROUVEL, Les corps de troupe de l'émigration française, $3 \mathrm{t}$. , Paris, La Sabretache, 1957-1964, t. 3, 405 p. : Armée des princes, armée de Bourbon, services de l'Autriche, de la Sardaigne, de l'Espagne et de la Suède, 1789-1815, p. 45 et 47.

41.Sydney CHURCHILL, Réimpression de l'état militaire de France [de Roussel] pour l'année 1789 [avec un index alphabétique et des notes biographiques, généalogiques et historiques], 10 t. , Carnac, 1913-1925, t. 2, p. 207-213 ; et Arch. Guerre, 7Yd 794. 42.Arch. Guerre, $\mathrm{Yb} 844$, fo 20 . Une ordonnance du 20 juillet 1780 ayant substitué cinq sous-lieutenants aux trois exempts de chacune de deux compagnies de gardes d'Artois, le chevalier de Crussol avait obtenu pour Marguerye une commission de lieutenantcolonel de cavalerie le 4 septembre 1781 ; Arch. Guerre : contrôle des gardes du comte d'Artois, Yb 77, et dossier personnel, 7Yd 2123.

43.L. HARTMANN, op. cit., p. 152.

44.AD Calvados, 4 L 7 : Affaire de Lecomte et du curé de Colleville.

45. Voir C. PEYRARD, loc. cit.

46.AD Calvados, 376 Edt 1.

47.C. PEYRARD, loc. cit., p. 225, a résumé les griefs exprimés à l'encontre de Marguerye dans cette requête par la formule « aristocrate sans scrupules ».

48.AD Calvados, 4 L 7.

49.Nous risquons cet emprunt à R. GRIFFITHS, op. cit., alors même que nous avons reconnu l'impossibilité de qualifier Marguerye de « monarchien ». Mais centriste ? 50.AD Calvados, 376 Edt 1.

51.Un certificat, obtenu par Marguerye le 31 août 1793 à Paris de l'adjoint de la 6e division du département de la Guerre, n'a pas été enregistré à Colleville, non plus que deux autres que nous citerons, provenant de la même source : AN, F7 5289, n 32764. Les certificats que renferme ce dossier ont étéproduits au Ministère de la Police après la loi d'amnistie du 17 messidor an VII (5 juillet 1799).

52.AD Calvados, $376 \mathrm{Edt} 1$. Les documents numérotés 4, 5, 7 et 8 contenus également dans le dossier du Ministère de la Police : AN, F7 5289, nº 32764.

53.Secteur resté en dehors de l'invasion.

54.« Au nom de la nation, je jure de maintenir de tout mon pouvoir la liberté et l'égalité, ou de mourir à mon poste ».

55.Chez Charles-Joseph-Victor Tuffin-Ducy, cousin germain maternel de Marguerye (cf. le baptême de sa fille Virginie en 1790).

56.Il existe plusieurs localités de ce nom dans l'Est de la France.

57.Le certificat ne tarit pas de louanges pour Marguerye. Or, l'identification des signataires d'août 1792 et de septembre 1793 a révélé qu'il s'agissait surtout d'officiers de fortune de l'Ancien Régime ou de sous-officiers récemment élevés en grade ; cf : État militaire de France, de RoUSSEL, pour les années 1782 à 1793 ; CHURCHILL, op. cit., t. 9, p. 217-230, et Xavier AUDouin, Service des officiers de tous grades, ou « Recueil de Xavier Audouin ", 3 vol. , Paris, 1793, vol. 3, p. 58-60 de la partie consacrée aux troupes à cheval. 58.Ce certificat peut avoir été produit en conformité avec un décret du 27 germinal an II (16 avril 1794) excluant les ex-nobles de Paris et des villes maritimes et fortifiées ; 
Louis RONDONNEAU, Collection générale des lois depuis 1789 jusqu'au 1er avril 1814, 16 t. , t. 4, 2e partie, Paris, 1818, p. 1062. La qualification de négociant, difficilement vérifiable quant à sa véracité, effaçait la noblesse, mais aussi justifiait les déplacements ; voir Patrice HIGONNET, Class, Ideology and the Rights of Nobles during the French Revolution, Oxford, 1981, 358 p. , p. 133, et sa note $\mathrm{n}^{\circ} 123$ p. 321.

59.Pierre SERNA, Antonelle, aristocrate révolutionnaire, 1747-1817, Paris, 1997, 499 p. , p. 190-193.

60.Par exemple, le certificat $n^{\circ} 7$ expose clairement qu'il est « fourni en exécution de la loi rendue contre les émigrés le 28 mars $1793 »$. Sur les étapes de cette législation, Marc BOULOISEAU, Étude del'émigration et de la vente des biens des émigrés (1792-1830), Paris, 1963, 179 p. , p. 77-91.

61. Mention dans un état des créances de Madame de Marguerye sur son mari : pièce jointe au contrat du 19 août 1822, AN, Minut. cent., XIV, 663.

62.AD Manche, magasin 5/2 (documents sur les émigrés) ; en août 1799, les bureaux du Ministère de la Police générale se référaient encore à cette inscription (AN, F7 5289, $n^{\circ} 32764$ ).

63. AD Manche, ibid.

64.C. PEYRARD, loc. cit., p. 226-227.

65.AD Calvados, 376 Edt 1.

66. Ce membre de la municipalité de Colleville était également fermier de Marguerye.

67.AN, F7 5289, n 32764.

68.L. RONDONNEAU, op. cit., t. 4, 2e partie, Paris, 1818, p. 606-607.

69. Christine LE BOZEC, La Normandie au XVIIIe siècle. Croissance, Lumières et Révolution,

Rennes, 2002, 222 p. , p. 185.

70.AD Calvados, 376 Edt 1 , et 376 Edt $3: 1810-1825$.

71.Résidant ordinairement à Valognes, celui-ci serait mort dans la capitale le 30 juin 1799, selon mention dans l'acte de mariage du fils de Bon-Henri-Marie à Paris du 13 juillet 1819 : Arch. Paris, V. 2 E.

72.AN, Minut. cent., XIV, 535, 21 prairial an III (9 juin 1795).

73.Il y vend en effet avec son épouse une des maisons appartenant à cette dernière : AN, Minut. cent., XLVII, 427, 29 thermidor an V (12 août 1797).

74.AN, Minut. cent., VII, 546, cautionnement du 27 nivôse an VII, (16 janvier 1799).

75. Notamment par la loi du 9 frimaire an VI (29 novembre 1797), consécutive au coup d'État anti-royaliste du 18 Fructidor : voir p. HIGONNET, op. cit., p. 234-243, sur cette loi et les motifs idéologiques qui l'auraient sous-tendue (et plus généralement son chapitre 6 sur la politique du Directoire à l'égard des nobles).

76.M. BOULOISEAU, op. cit., p. 90.

77.AN, F7 5289, n 32764.

78.Ibid. ; il est vrai que le patronyme Marguerye (ou Marguerie) était assez répandu en Basse Normandie. Léon DE LA SICOTIÈRE, Louis de Frotté et les insurrections normandes, 1793-1832, Paris, 1889, 3 t. de 629, 812 et 56 p. , passim, cite le chef chouan Marguerye, surnommé « Griffon », et, t. 2, p. 14, Henry de Marguerye, qui fit passer le confesseur de Louis XVI en Angleterre à partir du Bessin. Ajoutons un cousin de Charles, ÉdouardMarie Marguerye de Vierville, guillotiné le 31 mai 1794 (cf. AN, W 376, fo 67-76).

79.AN, F7 5289, nº 32764.

80.Jean-Claude MENES, La coalition du marquis de La Rouërie (1791-1792), thèse multigraphiée Rennes II, sous dir. Roger Dupuy, 2000, 445-13 p. , p. 83, 85-87, 125-126. 
Paul DELARUE, Charles-Armand Tuffin, marquis de La Rouërie, chef de la conjuration bretonne, Rennes, 1994 (fac-sim. de l'édition de 1899), II-223 p. , p. 28-29.

81.AN, F7 5289, nº 32764.

82.Jacques-Olivier BOUDON, Histoire du Consulat et de l'Empire, 1799-1815, réédition Paris, 2003, 512 p. , p. 97.

83.AD Indre, $P$ 740. Les AD Loire ne conservent (en P 4) que la prestation de serment de Marguerye du 4 nivôse an XII (26 décembre 1803). Pierre-François PINAUD, Les Receveurs généraux de 1800 à 1865. Une administration de transition, thèse sous dir. Louis Bergeron, EHESS, 1979, 376 p. dactyl., signale (p. 119) que, durant l'Empire, les fonctionnaires des finances ne furent plus obligés de prêterserment.

84. C'est en janvier 1806 que Mollien remplaça Barbé-Marbois.

85.AN, AF IV*345, p. 314 ; AF IV* 404, n² 254, et surtout AF IV 205, plaquette 1336, pièces 58 à 61 : minute du décret où le nom de Marguerye a été porté de la main de Napoléon.

86.Probablement du fait de la discrétion du patronyme (qui avait pu servir le Marguerye gyrovague).

87. Nous avons reconstitué la liste de ces neuf nobles à partir des indications de p. F. PINAUD, thèse citée., p. 189-195, et du même : Les Receveurs généraux des Finances, 1790-1865. Étude historique. Répertoire nominatif et territorial, Genève, 1990, 251 p. , passim.

88.Selon les indications de S. CHURCHILL, op. cit., t. 2, p. 205-216.

89. AD Indre, $\mathrm{P} 744$.

90.P. F. PINAUD, thèse citée, p. 122 pour le traitement, p. 247 et 260-262 pour le rôle officieux de banquiers des receveurs généraux.

91.AD Indre, p. 745. p. F. PINAUD, thèse citée, p. 262, note « que ces faillites en tant que banquier sont relativement rares, puisqu'en 65 ans d'histoire de la Recette, et sur plus de quatre cents fonctionnaires, il n'y eut que douze cas de faillites de Receveurs en tant que banquier $»$.

92.AD Indre, $P 745$.

93.AD Indre, 3 U 1/512.

94.AN, AF IV 475, plaquette 3618, pièces 72 et 71, et AF IV* 400, $\mathrm{n}^{\circ} 109$.

95.AD Indre, P 745.

96.S. CHURCHILL, op. cit., p. 207-213.

97.Dossier personnel : Arch. Guerre, 7Yd 794.

98.Signalons que la Restauration se sépara de vingt-quatre receveurs impériaux. Sur les neuf nobles d'Ancien Régime rencontrés plus haut, trois ne furent pas conservés ; cf.

p. F. PINAUD, thèse citée, p. 119.

99.Dossier personnel : Arch. Guerre, 7Yd 2123. Sur l'éphémère « régiment des chasseursimpériaux russes ", créé à Coblence le 17 février 1792, et dont le licenciement semble s'être produit dansl'évêché de Liège fin novembre, voir le vicomte GROUVEL, op. cit., t. 3, p. 123-127.

100.Dossier : Arch. Guerre, 7Yd 2123.

101. Voir le comte d'ESPINCHAL, Journal d'émigration, publié par Ernest d'Hauterive, Paris, 1912, 559 p. , en particulier p. 450-451 sur la « désorganisation à Arlon » à la mi-octobre 1792.

102.« Un des objectifs du mémorialiste est [...] de se réapproprier un destin qu'il n'a pas eu le sentiment de maîtriser ", écrit Karine RANCE dans « Mémoires de nobles français émigrés en Allemagne pendant la Révolution française : la vision rétrospective d'une 
expérience ", Revue d'histoire moderne et contemporaine, 1999, t. 46-2, p. 245-262 (p. 249

pour la citation).

103. Arch. Guerre, 7Yd 2123.

104.S. CHURCHILL, op. cit., p. 207-216 ; on y apprend que Gouvello ne reçut sa distinction qu'en 1827, treize ans après l'autre récipiendaire, le baron Mousin de Bernecourt.

105.AD Calvados, 376 Edt 3.

106.Ibid., 3 M 37-44.

107.AN, Minut. cent., XIV, 663, 19 août 1822.

108.AD Calvados, 3 M 47-75 : Listes des électeurs et du jury, 1829-1848.

109.Paul BASTID, Les institutions politiques de la monarchie parlementaire française

(1814-1848), Paris, 1954, 425 p. , p. 227.

110.AD Calvados, 3 M 51 et 63, contiennent des listes manuscrites de votants pour 1833 et 1839. Aucune signature d'un membre du bureau de vote recevant les bulletins de l'électeur n'accompagne le nom de Charles de Marguerye.

111.AM Bayeux, état civil.

112.AD Calvados, 8 E 6082 : notariat de Trévières, 17 février 1840, et 8 E 9992 : notariat de Bayeux, 24 juin 1840. L'épouse et les enfants déclarent ignorer les dettes du défunt, sauf en ce qui touche à la veuve.

113.AM Bayeux, état civil.

114.AD Calvados, 3 Q 1577.

115.P. SERNA, La République des girouettes, op. cit., p. 225.

116.Dictionnaire de girouettes [...], Paris, 3e éd. en 1815 (443, puis 491, puis 501 p. ) ;

Almanach des girouettes [...], Paris, 1815, $188 \mathrm{p}$.

117.AD Calvados, 376 Edt 3 à 7 : délib. mun. Colleville-sur-mer, 1810-1889.

118. Madame de Marguerye laissait une succession de 116000 francs ; AD Calvados, $8 \mathrm{E}$ 1000 7, notariat de Bayeux, 4 janvier 1844.

119.E. DE WARESQUIEL, L'histoire à rebrousse-poil, Paris, 2005, 190 p. , p. 143-144.

120.Nous tirons ce néologisme de la lecture de R. GRIFFITHS, op. cit., p. 10-11, définissant la« mitoyenneté "comme" idéologie inhérente » au centre, la modération étant conçue comme une fin plus qu'un moyen.

121.Dans le sens souhaité par p. SERNA, La République des girouettes, op. cit., p. 542.

122.C. LE BOZEC, op. cit., ch. III, « La Révolution en Normandie », p. 144-206, passim.

\section{RÉSUMÉS}

La jeunesse de Charles de Marguerye à la fin de l'Ancien Régime fut marquée par une accession à la maison militaire du comte d'Artois, donc à la cour, insolite dans son milieu. Au début de la Révolution, son action publique, mais surtout des opinions exprimées en privé, prouvent une volonté d'adaptation, ce qui ne lui évita pas des difficultés. Rallié à Bonaparte, il entama une carrière administrative qu'il ne parvint pas à poursuivre. Rallié aux Bourbons, il retrouva du crédit auprès du comte d'Artois, ce qui lui valut une retraite convenable. Le récit linéaire de cette vie n'aurait au mieux d'intérêt que relativement à des monographies du même type, s'il ne 
s'appuyait sur des documents peu fréquents : certificats des années 1792 à 1794, mais surtout lettres de 1791, ces dernières permettant de mettre en relation des opinions et des actes.

INDEX

Mots-clés : noble libéral, modéré, centre, girouette

\section{AUTEUR}

DIDIER MICHEL

21 bis rue Barillet-Deschamps

37000 Tours

didier.michel34@wanadoo.fr 